\title{
Wavelet Based Finger Knuckle and Finger Vein Authentication System
}

\author{
${ }^{1}$ Sujata Kulkarni and ${ }^{2}$ Ranjana Raut \\ ${ }^{1}$ Yeshwantrao Chavan College of Engineering, Nagpur; \\ RSTM University of Nagpur (India) \\ ${ }^{2}$ Electronics Department, SGM University Nagpur, India; \\ taresujata@yahoo.com; r24rd164@rediffmail.com
}

\begin{abstract}
Biometrics is the prominent technology for accurate and safe detection of claim identity. This paper proposes a novel multimodal authentication system using finger knuckle (FK) and finger vein (FV). Finger Knuckle has unique bending and makes this a distinctive biometric identifier. The vein pattern of all fingers of human being is not same. Each finger of same person has different vein pattern. It is the hidden part which is not seen by normal eye sight hence less possible to forge. The system consists of proposed prototype finger knuckle and finger vein image capturing devices, formation of own FK and FV image database acquired from proposed devices. Here feature extraction of FK images is based on Walsh Wavelet Transform and FV image on Hybrid Wavelet Transform. Proposed multimodal biometric authentications integrate transformed domain features vector of FK and FV at score level fusion using Bayesian and weighted sum method. The fusion of these two modalities using Bayesian method demonstrated the recognition accuracy of $98.3 \%$ and weighted sum $98.5 \%$. Various weights of finger knuckle and finger vein affects the recognition accuracy. The better recognition accuracy is obtained at weight of 0.8 and 0.2 . The performance index is improved i.e $98.5 \%$ and the Error equal rate is $1.5 \%$ as compare to unimodal biometric. Error equal rate is reduced by $10 \%$ than individual biometric system. For $\mathrm{N}$ user with $\mathrm{M} 1$ and $\mathrm{M} 2$ as test and training samples, for verification of one user, matching complexity is $\mathrm{O}(\mathrm{M} 1 \mathrm{M} 2)$ and for $\mathrm{N}$ user $\mathrm{O}(\mathrm{M} 1 \mathrm{M} 2 \times \mathrm{N})$. For identification, $(\mathrm{N} \times \mathrm{M} 1)$ test samples and $(\mathrm{N} \times \mathrm{M} 2)$ training samples are considered. So matching complexity is $\mathrm{O}[\mathrm{N}(\mathrm{N}-1) \times \mathrm{M} 1]$ for each biometric. Using conventional matching the complexity is $\mathrm{O}$ [N (N-1) x M1 M2]. For multimodal biometric using FK and FV, matching complexity is $\mathrm{O} 2[\mathrm{~N}(\mathrm{~N}-1) \times \mathrm{M} 1]$. It shows great reduction in matching complexity using the proposed algorithms.
\end{abstract}

Keywords: Finger knuckle, Finger vein, Wavelet, Hybrid Wavelet, Authentication, Fusion, Error Equal Rate, ROC curve

\section{Introduction}

Traditional authentication systems based on password, access card, lock and key etc. have a lot of issues of stolen the card, misuse of card, forgotten password and different password attacks. Today's wireless world, transaction through internet is demanding from the users hence needs to develop an authentication system that gives more security of individual identity. Modern authentication system based on human physiological and behavioural detail gives security to personal information and not easy to forge is known as biometric recognition system. It is generally accepted that physical traits like iris, fingerprints, finger knuckle, finger vein, DNA finger print [1] can uniquely defined each member 
Sujata Kulkarni and Ranjana Raut; Wavelet Based Finger Knuckle and Finger Vein Authentication System. Advances in I mage and Video Processing, Volume 4 No 4, August (2016); pp: 1-16

of large population which makes them suitable for large scale identification. Reason of attraction of such traits is social acceptance and easy to use. Finger knuckle (FK) is user centric, contactless and unrestricted access control. Texture and statistical features are available and easily extracted. It is independent to any behavioural aspect. No stigma of potential criminal investigation is associated with this approach [2]. Finger vein are internal part hence impossible to forge, unique, reliable, secure because not traceable by eye, less failure to enroll rate (FET), no issues of wet, dry, dirt like finger print. Proposed paper uses finger knuckle and finger vein as two biometrics for authentication. Unimodal biometric authentication is performed by identifying only single trait. Each biometrics system is not able to enroll all types of data. Failure to enroll is big issue in unimodal biometrics. Unimodal biometrics trait experiences a lot of problems such as noise data, spoof attacks, intra-class variation and unacceptable error rate. The advanced biometric concept known as multimodal biometric systems are used to overcomes the limitations of unimodel biometric systems. It integrates multiple evidences such as multiple units, multiple samples, and multiple traits from multiple sources of information [3]. Multimodal system can combine correlated as well uncorrelated biometric traits. Multimodal biometric is more reliable and secure due to use of multiple evidences. The integration of multiple evidences is possible using fusion techniques at different level [4]. Categories of multimodal biometric depend upon the integration of different basic units. Such system requires longer verification time thereby causing inconvenience to the users. Due to these limitations, the number of identifiers (modalities) in a multimodal biometric system is usually restricted to two or three.

The paper is organized as follows. Section 2 presents proposed multimodal system, Section 3 presents finger knuckle and FK database Section 4 describe finger vein acquisition devices and FV data base, Section 5 discuss feature vector generation using Kekre and Hybrid wavelet transform. Section 6 discusses fusion of two modalities. Results and conclusion are presented in Section 7.

\section{Proposed Multimodal Authentication System}

The Proposed system uses finger knuckle and finger vein as biometric traits and integrate transformed domain features extracted from them at matching score level. It mainly consists of three components: finger knuckle unit, finger vein unit and score level fusion unit. Finger knuckle recognition is responsible for matching the input finger knuckle against the finger knuckle templates stored in the database to obtain finger knuckle matching scores. Finger vein recognition is responsible for matching the input finger vein against the finger vein templates to obtain finger vein matching score. This score is normalized using Min-max normalization. Score level fusion is obtained using weighted sum and Bayesian rule. It integrates matching scores from finger knuckle and finger vein recognition block and forms the new scores which make the final decision. The framework of proposed multimodal biometric authentication system shows the integration of finger knuckle and finger vein at score level as shown in Figure1 




Figure 1: Framework of Proposed Multimodal System

\section{Proposed Finger Knuckle Acquisition Device}

Proposed prototype Finger knuckle capturing device is composed of light weight acrylic box with white background, finger supporter and digital camera (SonyDSC-W380) as shown in Figure 2. Camera captures the FK images with white and black background. The captured images with black background are darker than white background. Hence images with white background are used for reorganization.

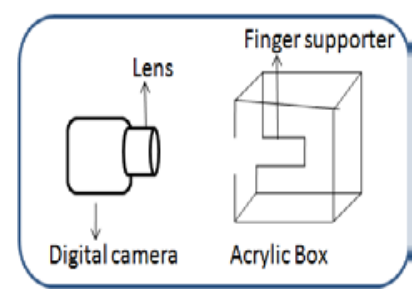

(a)

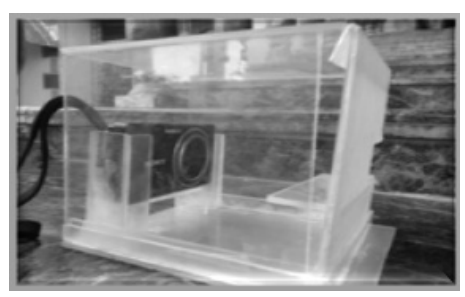

(b)

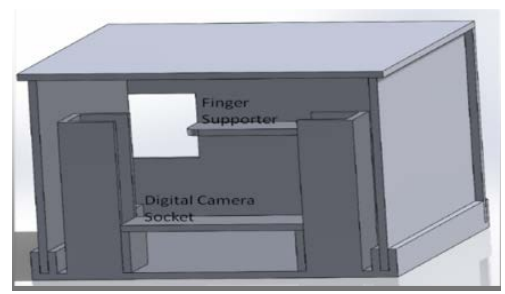

(c)

Figure 2: Finger knuckle acquisition device (a) prototype device (b) frame work of device (c) Modified FKP device [20]

During acquisition, user places finger from the notch at front side of device on finger supporter. Notch size is optimum, so user with any finger size can place finger on supporter and FK images can be acquired. During FK images acquisition, translation or rotations of finger take place. Such movement can increase the variation in finger knuckle feature of authenticate user. To overcome these limitations, device uses folding bar as guiding structure for finger tips and part of back side of finger. Finger bracket is designed for this purpose. The modification in capturing device is made with proper design using Solid Works 2013.

- In modified device, finger notch is flexible and mounted on the finger supporter.

- Modified FKP device is compact in size (140 mm x $130 \mathrm{~mm} \times 90 \mathrm{~mm})$, cost effective, user friendly and acquire FK images with high resolution ( $4300 \times 3200$ pixels).

- Distance between the camera and knuckle is $89 \mathrm{~mm}$ and with white back ground.

- The distance between the camera and supporter is $89.04 \mathrm{~mm}$

- Height of the device is reduced to $90 \mathrm{~mm}$ to avoid outside reflection during acquisition of FK images.

The proposed proto type finger knuckle acquisition device survives under uncontrolled lighting conditions and deformation due to finger orientation and perspective projection. Hence, users of the biometric authentication system can be relieved from the careful planning of acquisition environment 
and unnatural hand orientation during capture. The FK images used in proposed system are acquired by ordinary cameras equipped on portable devices.

\subsection{Finger knuckle image database}

As per hand geometry, number of finger is available for acquisition. Proposed system indented to capture only the middle knuckle of right index and right middle finger. Among five fingers, lower, middle and upper knuckles are present only on the four fingers except thumb. Small finger knuckle is too small in area. Database consists of finger knuckle of right index and middle knuckle of all classes to cover the entire population. FK image database consists of 50 users (18 to 68 years) from an educational institute including different categories such as VIP, teaching, non-teaching, students and workers. To consider variant finger knuckle location and orientation, FK images are acquired in two phases with average interval of days and time. By using proposed image acquisition devices, Images from the same finger collected at different time are similar to each other while images from different fingers are different; this implies that FKP has potential for personal identification. Figure 3 shows some of sample FK images acquired from the proposed device.

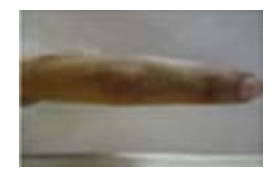

User1_1index...

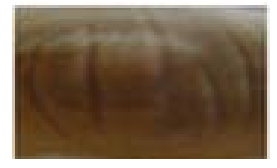

User1_1index..

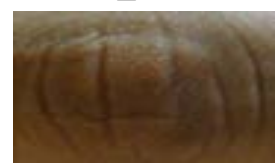

User1_1index...

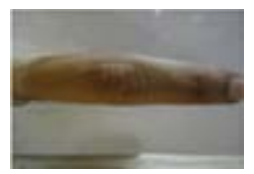

User1_5index

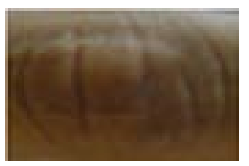

User1_5index

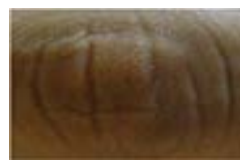

User1_5index



User1_1 middle...

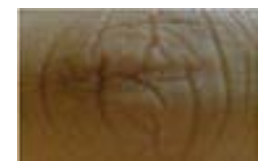

User1_1 middle...



User1_1 middle...



User1_5middle

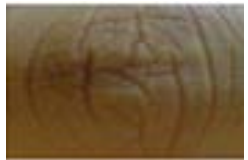

User1_5middle



User1_5middle

Figure 3: Some of sample finger Knuckle images acquired from proposed prototype acquisition device(a)Raw FK (b) Cropped FK (c) Resized FK samples [20]

\section{Proposed Finger Vein acquisition Device}

Proposed FV image acquisition device consists of LEDs assembly, capturing unit, power supply unit, and display. Finger vein acquisition device is based on NIR optical source and simple IR camera to capture the vein pattern. NIR assembly consists of series alignment of NIR LEDs of $750 \mathrm{~nm}$ non-contact with finger as shown in figure 4. Numbers of LEDs are chosen to cover whole finger of any person as thickness of finger changes person to person such as fat, medium and thin finger. Finger is placed on NIR assembly. When power in ON, light is radiated from the NIR LEDs passes through the finger and is absorbed by haemoglobin of blood and shows dark blood vessel pattern. This pattern is captured by IR camera. Simple webcam without IR filter act as IR camera. NIR imaging is safe because it penetrate only the superficial area of the finger. When light is radiated in the finger, temperature of the skin increases, but it is controlled by adjusting operational parameter of LED that make LED within safe temperature range [10]. In this system, finger is in non-contact with NIR LEDs and acquisition of vein is in milliseconds hence safer. Here IR camera is installed on laptop. Driver of laptop are changed because webcam is inbuilt in laptop which senses the visible light while IR camera senses the IR light. 
If the display screen is desktop, then driver are not changed. The physical set up finger vein acquisition is as shown in figure 5

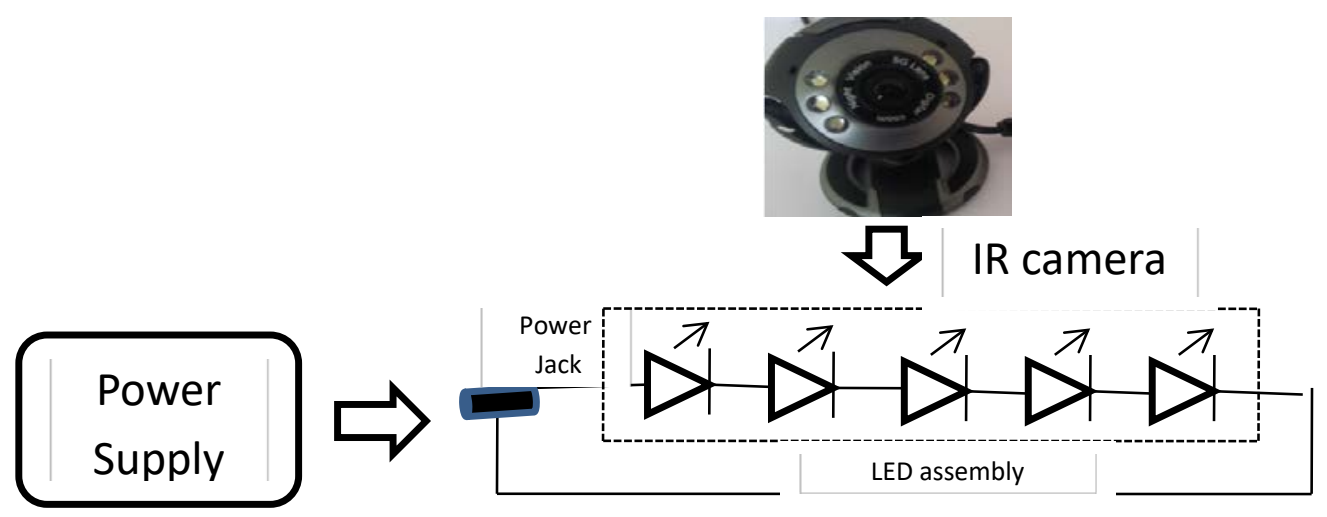

Figure 4: Optical source and camera set up of Finger vein device [21]

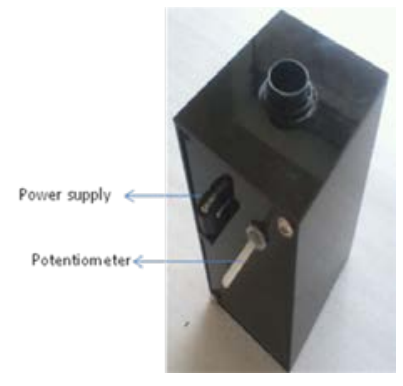

(a)

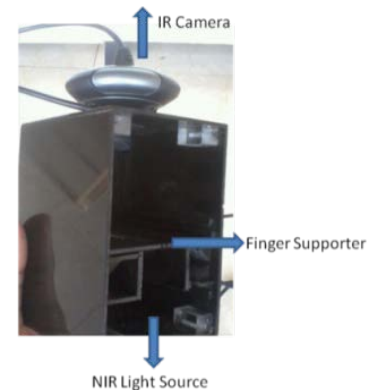

(b)

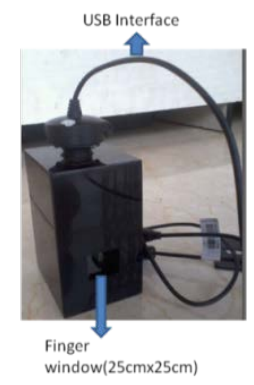

(c)

Figure 5: Physical set up of Finger Vein Device (a) Back view (b) Internal framework (c) Font view [21]

\subsection{Finger Vein Image database}

Total 50 users are considered during enrolment procedure. Ten samples of each unit are taken so total 20 samples of each user. Database of 50 users is formed using 500 finger vein samples at different orientation. Database covers different categories like VIP person to worker class users and senior citizen. It is further classified as gender and age. Users of age of 18 to 65, fatty, weak and moderate health persons are involved. Finger vein samples of users with different categories are taken in order to analyze the failure to enrolled rate. It is noted that, three user could not enroll finger vein samples due to weakness whose haemoglobin level is not up to the mark. Another two users are not able to enroll due to their natural finger style. Alignment of vein pattern is not proper because of natural bend at first knuckle. Sample finger vein image are as shown in figure 6 . The captured raw finger vein samples are of size $640 \times 480$ as shown in figure 6 (a) and (b). From the original vein samples middle part of fingers vein are cropped to obtain region of interest with finger geometry.

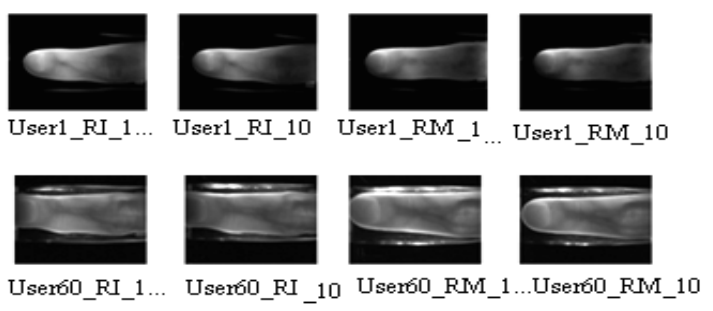

(a) 


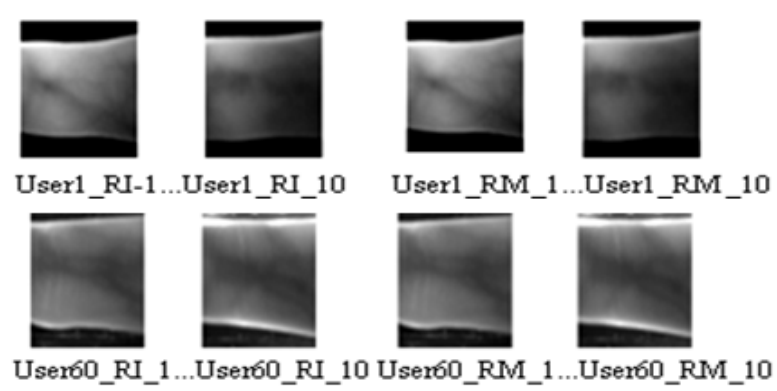

(b)

Figure 6: Acquired Finger vein pattern (a) Raw finger vein pattern (b) cropped Finger vein samples Finger knuckle and Finger vein Enhancement

Images captured from the modified FK device are raw images. These images are enhanced to get better clarity of unique features such as ridges, creases around the phalangeal joint of finger knuckle surface. This is very important step to improve the recognition. We use Weiner filter and reflection removal as pre-processing step to enhance the quality of FK images. Images with many edges are handled by local wiener filter. Hence de noising all the FKP is done using Winner filter [17] .Original images has curvature surface hence results in non-uniform reflection. To obtain well distributed texture, we use the reflection removal technique [15]. The reflection filtered image is enhanced image from which feature are extracted. Features are extraction using Kekre Wavelet Transform. During the enrolment stage, the raw vein pattern shows noise due to high temperature and transmission noise created by the IR radiation. Actual features of vein pattern get hide due to such noise. Hence need to apply the pre-processing method to get better clarity from the raw samples.

This paper explores the bilateral and median filtering techniques for noise removal. Median filter is used to remove noise in the form of defective pixel and make the samples noise free. Edge preservation is another property of median filter which is the important information for vein samples [19]. But edge retention using median is not applicable for all condition hence second de noising bilateral technique is used. Contrast adaptive histogram reduces amplified noise by clipping the histogram. Histogram is clipped at specific value called as clip limit. Generally amplification can be limited by common value between 3 and 4.The amplified part beyond the clip limit is uniformly redistributed among all histogram $[11,15]$ and get enhanced vein pattern.

\section{Feature Vector Extraction of Finger Knuckle}

Walsh is an orthogonal transform, so Walsh Wavelet is generated from Walsh transform [118]. $16 \mathrm{x}$ 16 Walsh Wavelet from $4 \times 4$ Walsh transform matrix as shown in Figure 7. 


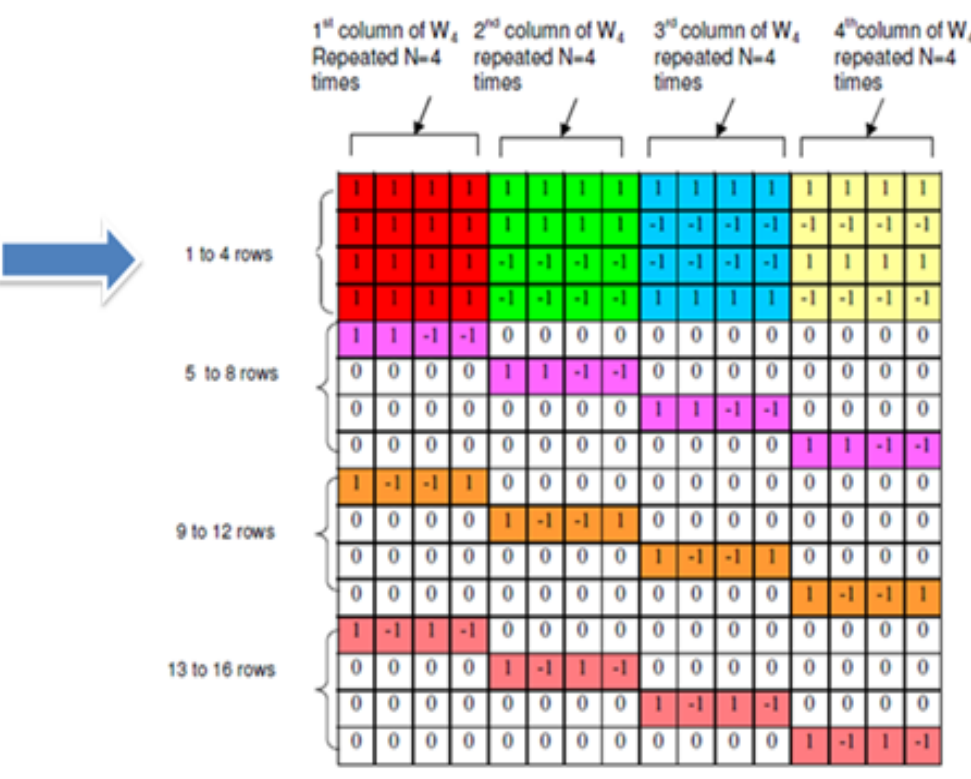

Figure 7: Walsh wavelet transform

The ROI is selected to be of size $128 \times 256$. The Walsh wavelet transform of size $128 \times 128$ is selected. Extract all the features of knuckle from the flow shown in figure 8 . These features are the energy coefficient calculated by following equation 1 .

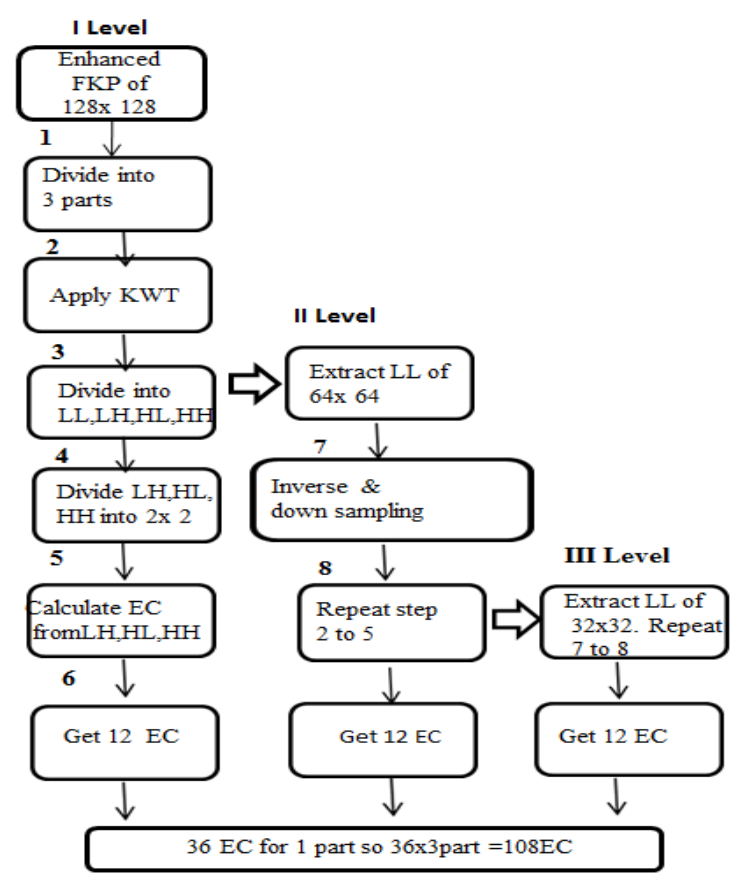

Figure 8: Flow of Feature extraction using WWT

$$
W E_{L C}=\sum_{i=0}^{w-1} \sum_{j=0}^{w-1} W C(i, j)^{2}
$$

Where $L=0,1,2 ; C=0(\mathrm{LH}), 1(\mathrm{HL}), 2(\mathrm{HH})$ and $W$ is the size of wavelet component in the different level i.e. $64,32,16$ respectively. Similarly a set of 4 wavelet coefficients are obtained from HL and HH. The values of these 3 set of coefficients give 12 features from the first level, 12 from second and 12 from third so total 36 energy coefficients for left part of enhanced sample. The same procedure is carried out on right and centre block. Hence, the final feature vector has 108 feature values. The values of the feature vector are taken without normalization. Such feature vectors are generated for training 7 

Advances in Image and Video Processing, Volume 4 No 4, August (2016); pp: 1-16

samples of each user and over database of 50 users. Therefore, the training samples are 350 . A matrix file of all the feature vector of 350 samples is created.

\subsection{Feature Vector Extraction of Finger Vein}

Hybrid Wavelet Transform is used for combining the traits of two different orthogonal transform wavelets to exploit the strengths of both transform wavelets. The hybrid wavelet transform matrix of size $\mathrm{N} \times \mathrm{N}$ (say ' $T A \mathrm{~B}^{\prime}$ ) can be generated from two orthogonal transform matrices (say $\mathrm{A}$ and $\mathrm{B}$ respectively) with sizes $\mathrm{p} \times \mathrm{p}$ and $\mathrm{q} \times \mathrm{q}$. where $\mathrm{N}=\mathrm{p} * \mathrm{q}=\mathrm{pq}$. Hybrid transform matrix is generated from matrix $A$ and $B$. Proposed finger vein recognition system developed the hybrid wavelet using Kekre and DCT for global feature extraction as represented in figure 9.

$A=$\begin{tabular}{|c|c|c|c|}
\hline$a_{11}$ & $a_{12}$ & $j$ & $a_{1 p}$ \\
\hline$a_{21}$ & $a_{22}$ & $\cdots$ & $a_{2 p}$ \\
\hline$\vdots$ & $\vdots$ & $\cdots$ & $\vdots$ \\
& & $\cdot$ & \\
\hline$a_{p 1}$ & $a_{p 2}$ & $\cdots$ & $a_{p p}$ \\
\hline
\end{tabular}

Kekre Transform Matrix

$\mathbf{B}=$\begin{tabular}{|c|c|c|c|}
\hline $\mathbf{b}_{11}$ & $\mathbf{b}_{12}$ & $\cdots$ & $\mathbf{b}_{1 \mathrm{q}}$ \\
\hline $\mathbf{b}_{21}$ & $\mathbf{b}_{22}$ & $\cdots$ & $\mathbf{b}_{2 \mathrm{q}}$ \\
\hline$\vdots$ & $\vdots$ & $\cdots$ & $\vdots$ \\
& & & \\
\hline $\mathbf{b}_{\mathrm{q} 1}$ & $\mathbf{b}_{\mathrm{q} 2}$ & $\cdots$ & $\mathbf{b}_{\mathrm{qq}}$ \\
\hline
\end{tabular}

DCT Matrix

\begin{tabular}{|c|c|c|c|c|c|c|c|c|c|c|c|c|}
\hline $\begin{array}{l}a_{11} \\
b_{11} \\
b_{21} \\
\vdots \\
b_{q_{1}}\end{array}$ & $\begin{array}{l}a_{12} \\
b_{11} \\
b_{21} \\
\\
b_{q_{1}}\end{array}$ & . & $\begin{array}{l}a_{1 p^{*}} \\
b_{11} \\
b_{21} \\
\vdots \\
b_{q 1}\end{array}$ & $\begin{array}{l}a_{11} \\
b_{12} \\
b_{22} \\
\vdots \\
b_{42} \\
\end{array}$ & $\begin{array}{l}a_{12} * \\
b_{12} \\
b_{22} \\
\vdots \\
b_{q_{2}}\end{array}$ & $\cdots$ & $\begin{array}{c}\mathrm{a}_{1 \mathrm{p}^{*}} \mathrm{~b}_{12} \\
\mathrm{~b}_{22} \\
\\
\mathrm{~b}_{\mathrm{q} 2}\end{array}$ & $\ldots$ & $\begin{array}{cc}a_{11} * b_{1 q} \\
b_{2 q} & \vdots \\
& b_{q q}\end{array}$ & $\begin{array}{l}a_{12 *} \\
b_{1 q} \\
b_{2 q} \\
\vdots \\
b_{q q}\end{array}$ & $\ldots$ & $\begin{array}{l}a_{1 p^{*}} \\
b_{1 q} \\
b_{2 q} \\
\vdots \\
b_{q q}\end{array}$ \\
\hline$a_{21}$ & $a_{22}$ & $\ldots$ & $a_{2 p}$ & 0 & 0 & $\ldots$ & 0 & $\ldots$ & 0 & 0 & $\ldots$ & 0 \\
\hline 0 & 0 & $\ldots$ & 0 & $a_{21}$ & $a_{22}$ & 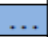 & $a_{2 p}$ & $\ldots$ & 0 & 0 & 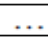 & 0 \\
\hline$\vdots$ & $\vdots$ & $\vdots$ & $\vdots$ & $\vdots$ & $\vdots$ & $\vdots$ & $\vdots$ & $\ldots$ & $\vdots$ & $\vdots$ & $\vdots$ & $\vdots$ \\
\hline 0 & 0 & $\ldots$ & 0 & 0 & 0 & $\ldots$ & 0 & $\ldots$ & $a_{21}$ & $a_{22}$ & $\ldots$ & $a_{2 p}$ \\
\hline$a_{31}$ & $a_{32}$ & $\ldots$ & $a_{3 p}$ & 0 & 0 & $\ldots$ & 0 & & 0 & 0 & $\ldots$ & 0 \\
\hline 0 & 0 & $\ldots$ & 0 & $a_{31}$ & $a_{32}$ & $\ldots$ & $a_{3 p}$ & & 0 & 0 & $\ldots$ & 0 \\
\hline$\vdots$ & $\vdots$ & $\vdots$ & $\vdots$ & $\vdots$ & $\vdots$ & $\vdots$ & $\vdots$ & $\ldots$ & $\vdots$ & $\vdots$ & $\vdots$ & $\vdots$ \\
\hline 0 & 0 & $\ldots$ & 0 & 0 & 0 & $\ldots$ & 0 & & $a_{31}$ & $a_{32}$ & $\ldots$ & $a_{3 p}$ \\
\hline$\vdots$ & $\vdots$ & $\vdots$ & $\vdots$ & $\vdots$ & $\vdots$ & $\vdots$ & $\vdots$ & $\ldots$ & $\vdots$ & $\vdots$ & $\vdots$ & $\vdots$ \\
\hline$a_{p 1}$ & $a_{p 2}$ & $\ldots$ & $a_{p p}$ & 0 & 0 & $\ldots$ & 0 & & 0 & 0 & $\ldots$ & 0 \\
\hline 0 & 0 & $\ldots$ & 0 & $a_{p 1}$ & $a_{p 2}$ & $\ldots$ & $a_{p p}$ & & 0 & 0 & $\ldots$ & 0 \\
\hline$\vdots$ & $\vdots$ & $\vdots$ & $\vdots$ & $\vdots$ & $\vdots$ & $\vdots$ & $\vdots$ & $\ldots$ & $\vdots$ & $\vdots$ & $\vdots$ & $\vdots$ \\
\hline 0 & 0 & $\ldots$ & 0 & 0 & 0 & $\ldots$ & 0 & & $a_{p 1}$ & $a_{p 2}$ & $\ldots$ & $a_{p p}$ \\
\hline
\end{tabular}

Figure 9: Hybrid Wavelet Transform from Kekre and DCT

Here, wavelet energy feature (WEF) is used to describe the vein texture. Hybrid Wavelet (HW) Transform of the selected vein pattern ROI is taken. The wavelets will capture localized spectral information from the ROI. Use only ROI of size $128 \times 128$ pixels. At each level, Mth order Hybrid Wavelet matrix ( $M$ X M Size) is generated by M/4 (64X64) order Kekre Transform Matrix [24.Figure 10 shows 1st level decomposition using Hybrid Wavelets. Take $128 \times 128$ finger vein Image and the first level The next level of decomposition is on matrix of size $N / 2 \times N / 2$ (half of the previous). This will continue $\mathrm{K}$ times for $\mathrm{K}$ level decomposition. In case of Hybrid wavelets there is a problem, as the $\mathrm{LL}$ components has size less than N/2 (or M in case of MP X MP size wavelet). Next level decomposition becomes difficult as at every level the size is reduced by $1 / \mathrm{P}-1$ factor, as well as the down sampling is also difficult. 
The proposed a novel method to overcome this issue discussed as follows. Instead of taking the LL component form the Hybrid Wavelet; take low Frequency component generated by other wavelets like Kekre Wavelet. As these wavelets have N/2 size component and generate separate set of Hybrid wavelet for each decomposition level shown in Table 1

Table 1: Wavelet Coefficients blocks for Wavelet Energy Features

\begin{tabular}{|c|c|c|c|}
\hline $\begin{array}{c}\text { Level } \\
\text { (Image Size } \\
\text { 128*128) }\end{array}$ & $\begin{array}{c}\text { Kekre } \\
\text { Wavelet LL } \\
\text { Component Size }\end{array}$ & $\begin{array}{c}\text { Hybrid } \\
\text { Wavelet LL } \\
\text { Component Size }\end{array}$ & $\begin{array}{c}\text { Parameters } \\
\text { used for WEF } \\
\text { Generation using } \\
\text { Hybrid Wavelets } \\
\text { (M,P) }\end{array}$ \\
\hline $\mathrm{K}=1$ & $\mathrm{NA}$ & $32 * 32$ & $\mathrm{M}=32, \mathrm{P}=4$ \\
\hline $\mathrm{K}=2$ & $64 * 64$ & $16 * 16$ & $\mathrm{M}=16, \mathrm{P}=4$ \\
\hline $\mathrm{K}=3$ & $32 * 32$ & $8 * 8$ & $\mathrm{M}=8, \mathrm{P}=4$ \\
\hline $\mathrm{K}=4$ & $16 * 16$ & $4 * 4$ & $\mathrm{M}=4, \mathrm{P}=4$ \\
\hline
\end{tabular}

Now, to generate the multi resolution analysis of the given Image data, here consider initial vein sample size as $128 \times 128$ Pixels.



Figure 10: Transformed finger vein pattern using KWT and HWT

LH



HL

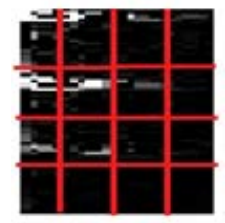

$\mathrm{HH}$



Figure 11: Energy coefficients from the Hybrid wavelet sample

$$
\begin{gathered}
E_{i}^{h}=\sum_{x=1}^{M} 1 \sum_{y=1}^{N}\left(H_{i}(x, y)\right)^{2} \\
E_{i}^{v}=\sum_{x=1}^{M} 1 \sum_{y=1}^{N}\left(V_{i}(x, y)\right)^{2} \\
E_{i}^{d}=\sum_{x=1}^{M} 1 \sum_{y=1}^{N}\left(D_{i}(x, y)\right)^{2}
\end{gathered}
$$


1. Take Original Image and evaluate first level decomposition using Kekres Wavelets and Hybrid Wavelets. Use LH, HL, \& HH Components of Hybrid Wavelets for generation of Wavelet Energy Distribution as per equations

2. Use LL component of Kekre Wavelet and generate next level input using Inverse Wavelet \& down sampling. This will be used as input for $\mathrm{K}=2$.

3. Using the input generated from Kekre Wavelet, perform Hybrid Wavelet Decomposition. For this level $(K=2)$ the size of input image component is $64 \times 64$. Generate the Hybrid Wavelet by $M=16 \& P=4$ and form the feature vector as discussed above.

4. Repeat Steps $3 \& 4$ for next levels and use parameters as calculated in Table 4.6 as per the procedure discussed. This procedure is shown for two levels $K=1 \& K=2$.

Using the above mentioned procedure the feature vector is generated, the feature vector has total 144 coefficients for $K=3(16$ X $3 \times 3=144,16$ Coefficient Per Components X 3 Component Per Level X 3 Levels of Components). Every decomposition level has reduced component size, hence the overall wavelet component energy goes on reducing and the value of feature vector coefficients goes on diminishing. Hence, the Feature Vector is normalized Each Level wise. Figure 6 shows the feature vector normalized level wise, here we can see the energy distribution. Three sections can be seen for five decomposition levels $(K=3)$. This feature vector is used for matching of the vein pattern. These feature vector is matching with the enroll samples of finger knuckle and finger vein is matched with Euclidean distance to generate a score.

\section{Score Level Fusion using Bayesian Method}

Let $w_{1}, w_{2} \ldots w_{n}$ represents $N$ users enrolled in the database. Let $\mathbf{x}$ be the feature vector corresponding to the primary biometric. Without loss of generality, let us assume that output of the primary biometric system is of the form

$$
P\left(\mathbf{w}_{\mathbf{i}} \mid x\right), i=1,2, \cdots, n
$$

where $\mathrm{P}\left(\mathrm{W}_{\mathrm{i}} \mid \mathbf{x}\right)$ is the probability that the test user is $\mathrm{W}_{\mathrm{i}} \mid$ given the feature vector $\mathrm{X}$. If the output of the primary biometric system is a matching score, it is converted into posterior probability using an appropriate transformation.

For the secondary biometric system, we can consider $P\left(\mathrm{~W}_{\mathrm{i}} \mid \mathbf{x}\right)$ as the prior probability of the test user being user $\omega_{\mathbf{i}}$.

Let $\mathbf{y}=\left[y_{1}, y_{2}, \ldots, y_{k}, y_{k+1}, y_{k+2}, \ldots y_{m}\right]$ be the second biometric feature vector, where $y_{1}$ through $y_{k}$ are continuous variables and $y_{k+1}$ through $y_{m}$ are discrete variables. The final matching probability of user $\mathrm{w}_{\mathrm{i}}$, given the primary biometric feature vector $\mathrm{X}$ and the second biometric feature vector $\mathrm{Y}$, i.e., $\mathrm{P}\left(\mathrm{w}_{\mathrm{i}} \mid \mathrm{X}, \mathrm{y}\right)$ is calculated using the Bayesian rule as [22]

$$
P\left(\mathrm{w}_{\mathrm{i}} \mid x, y\right)=\frac{p\left(y \mid \mathrm{w}_{\mathrm{i}}\right) P\left(\mathrm{w}_{\mathrm{i}} \mid x\right)}{\sum_{i=1}^{n} p\left(y \mid \mathrm{w}_{\mathrm{i}}\right) P\left(\mathrm{w}_{\mathrm{i}} \mid x\right)}
$$

Where $\mathrm{X}=$ input image feature vector

$\left.\mathbf{P}\left(\mathbf{w}_{\mathbf{i}} \mid \mathbf{x}\right)\right)=$ Euclidean distance in terms of probability (Primary finger knuckle biometric)

$\left.\mathbf{P}\left(\mathbf{y} \mid \mathbf{w}_{\mathbf{i}}\right)\right)$ = Euclidean distance in terms of probability (Secondary finger vein biometric) 
Theses Euclidean distances are converted in probability form in order to fuse at score level in the range of 0 to 1

$\mathbf{P}\left(\mathbf{w}_{\mathbf{i}} \mid \mathbf{x}\right)$ ) is the probability (FK) of user given the primary biometric,

$\mathbf{P}\left(\mathbf{y} \mid \mathbf{w}_{\mathbf{i}}\right)$ ) Where $\mathrm{y}$ is the probability (FV) of user given the identity claimed by the user. The scores of both primary and secondary biometric are normalized by Min-max normalization. Post score normalization, normalized average score is calculated and considered as the new score for multimodal identification system. If the correlation of claim user is less than new score, it is accepted else it is rejected from the decision module

Given a set of matching distance of finger knuckle recognition are $\left\{\boldsymbol{d}_{f k}\right\}$, for $k=1,2,3 \ldots, \mathrm{n}$, the normalization scores are given by,

$$
S_{\mathrm{fk}}=\frac{\mathrm{d}_{\mathrm{fk}-\min }}{\max -\min }
$$

Where $\boldsymbol{d}_{f k}, \boldsymbol{S}_{f k}$ are matching distances and matching score after normalization of finger knuckle respectively. Similarly $\boldsymbol{d} \boldsymbol{f} \boldsymbol{v}_{\boldsymbol{v}}, \boldsymbol{S}_{\boldsymbol{f v}}$ are the matching distances and matching score after normalization of finger vein biometric calculated using following equation as [feig].

$$
S_{f v}=\frac{d_{f v-m i n}}{\max -\min }
$$

The fusion method is tested on own finger knuckle and finger vein database consisting of 500 samples each with 10 samples of each user and each trait. So in total 1000 finger knuckles and finger veins are considered. The experimental test has shown the great improvement in the recognition accuracy of multimodal over individual biometric system which is represented in terms of TAR Vs. TRR and threshold graph as shown in Figure12.

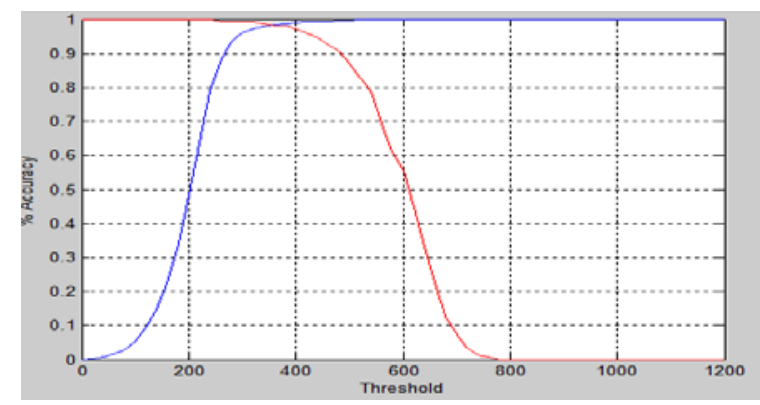

(a)

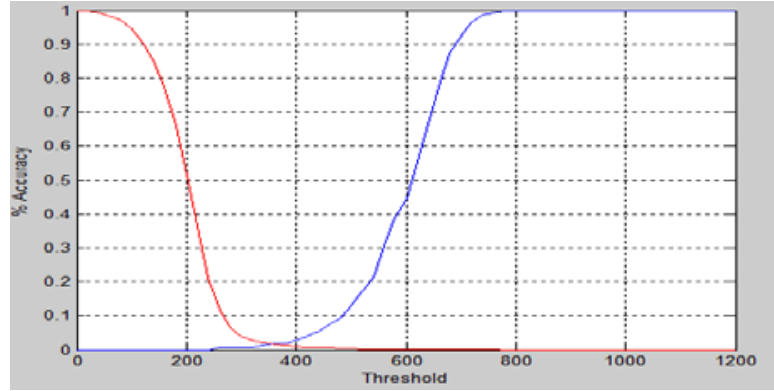

(b)

Figure 12: Recognition accuracy using Bayesian method

The graphical representation shows that the true acceptance rate increases using Bayesian fusion method. So indirectly the Error equal rate (EER) reduces as compared to the individual finger knuckle and finger vein. Table 2 shows the recognition accuracy of finger knuckle and finger vein using Bayesian method. 
Table 2: Recognition Accuracy of Multimodal system using Bayesian method

\begin{tabular}{|c|c|c|}
\hline \multirow{2}{*}{ Biometric } & \multicolumn{2}{|c|}{ Fusion Recognition Rate (\%) } \\
\cline { 2 - 3 } & TAR & FAR \\
\hline Finger knuckle (WWT) & 88.67 & 11.33 \\
\hline Finger Vein(HWT) & 88.22 & 11.88 \\
\hline Score Fusion & 98.3 & 1.7 \\
\hline
\end{tabular}

\subsection{Score Level Fusion using Weighted Sum Method}

Matching algorithms for finger knuckle and finger vein based recognition system are different. In both the cases, Euclidean distances are used for matching results of finger knuckle and finger vein recognition. Therefore, matching results scores of finger vein recognition are normalized. The Minmax normalization [6] simplest technique is used. The matching score obtained from Hybrid Wavelet Transform and Walsh Wavelet Transform are fused at score level. The fusion is done using weighted sum technique.

Let $\mathbf{W} \boldsymbol{f} \boldsymbol{k}$ and $\boldsymbol{W}_{f v}$ are weights of finger knuckle (WWT) and finger vein (HWT) respectively. The weights are given by

$$
\begin{gathered}
W_{f k}=\frac{c_{f k}}{c_{f k}+c_{f v}} \\
W_{f v}=\frac{c_{f v}}{c_{v}+c_{f b}}
\end{gathered}
$$

Where $\boldsymbol{C}_{f k}=($ TAR $/$ total images $) * 100 ;$ for Walsh wavelet transform

$$
C_{f v}=(\text { TAR / total images }) * 100 ; \text { for Hybrid wavelet transform }
$$

$\boldsymbol{M}_{f k}$ and $\boldsymbol{M}_{f v}$ are matching score of finger knuckle (WWT) and finger vein (HWT) respectively. The final score obtained after fusion [22] is as follows

$$
S=W_{f k} M_{f k}+W_{f v} M_{f v}
$$

Here $\boldsymbol{W} \boldsymbol{f} \boldsymbol{k}$ and $\boldsymbol{W}_{\boldsymbol{f v}}$ are their weights is the fusion score. The weights $\boldsymbol{W} \boldsymbol{f k}$ and $\boldsymbol{W}_{\boldsymbol{f v}}$ are varied over the range $[0,1]$, such that the constraint $\mathbf{W} f \boldsymbol{k}+\boldsymbol{W}_{f v}+=1$ is satisfied [22].

Table 3 presents the recognition accuracy of fusion at different weights of finger knuckle and finger vein. Here, weight for finger knuckle is the range $(0.9-0.5)$ and finger vein in the range $(0.1-0.5)$ are changed such that constraint $\mathbf{W} f \boldsymbol{k}+\boldsymbol{W}_{\boldsymbol{f v}}=1$ is satisfied.

Table 3 presents the recognition accuracy of fusion at different weights of finger knuckle and finger vein. Here, weight for finger knuckle is the range $(0.9-0.5)$ and finger vein in the range $(0.1-0.5)$ are changed such that constraint $+=1$ is satisfied.

It is observed that for recognition accuracy using multimodal recognition system is in the range of 96.5 $\%$ to $97.5 \%$ depending on the weight used for finger knuckle and finger vein traits. For finger knuckle weight in the range of 0.7 to 0.8 , system has maximum recognition accuracy of $98.3 \%$ to $98.5 \%$. For equal weight, recognition accuracy is $97.5 \%$. The recognition accuracy of unimodal system using finger knuckle and finger vein by using different algorithms is in the range of $90 \%$. The recognition accuracy 
of unimodal system using finger knuckle and finger vein by using different algorithms is in the range of 90 to $93 \%$.

Table 3 Recognition Accuracy of Multimodal system using Bayesian method at different weights

\begin{tabular}{|c|c|c|}
\hline$W_{f k}$ & $W_{f v}$ & $\begin{array}{c}\text { Fusion Recognition } \\
\text { Rate (\%) }\end{array}$ \\
\hline 0.9 & 0.1 & 96.5 \\
\hline 0.8 & 0.2 & 98.5 \\
\hline 0.7 & 0.3 & 98.3 \\
\hline 0.6 & 0.4 & 97.3 \\
\hline 0.5 & 0.5 & 97.5 \\
\hline
\end{tabular}

Figure 13 shows the performance of multi modal recognition system in terms of TAR Vs TRR using the weighted sum rule. Table 4 presents the recognition accuracy of multimodal system using score fusion level

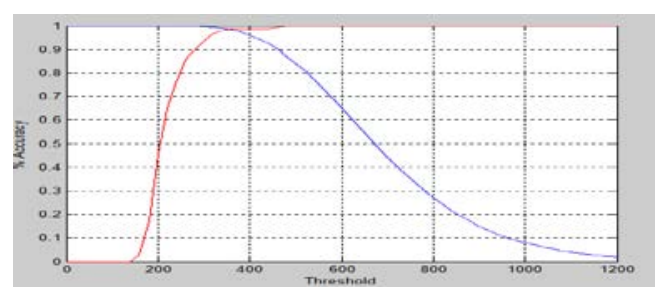

(a)

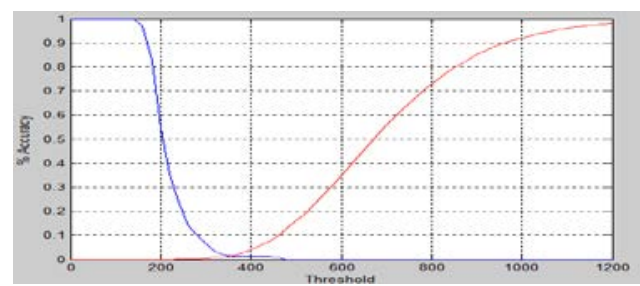

(b)

Figure 13: Recognition accuracy of multimodal biometric system using weighted sum

Table 4. Comparison of Recognition accuracy of multimodal system

\begin{tabular}{|c|c|c|}
\hline \multirow{2}{*}{$\begin{array}{c}\text { Multimodal } \\
\text { Biometric }\end{array}$} & \multicolumn{2}{|c|}{$\begin{array}{c}\text { Fusion Recognition Rate } \\
\text { performance (\%) }\end{array}$} \\
\cline { 2 - 3 } & TAR & FAR \\
\hline Weighted Sum & 98.5 & 1.5 \\
\hline Bayesian & 98.3 & 1.7 \\
\hline
\end{tabular}

The ROC plot is a graphical representation of Genuine Acceptance Rate (GAR) and False Acceptance Rate (FAR). Matching decision depends upon the threshold value that shows similar or correlates the claim template to stored template. ROC curve of figure 14 shows the comparison of score fusion

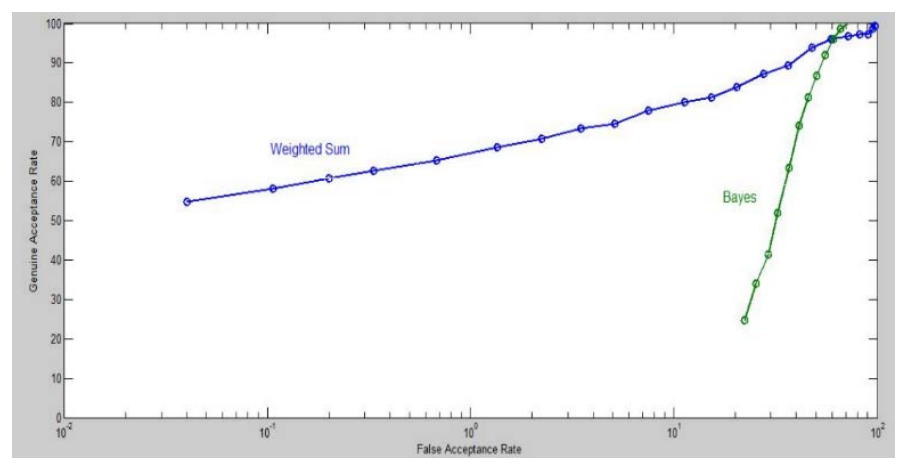

Figure 14: ROC Curve for score fusion 
Sujata Kulkarni and Ranjana Raut; Wavelet Based Finger Knuckle and Finger Vein Authentication System. Advances in Image and Video Processing, Volume 4 No 4, August (2016); pp: 1-16

ROC curve shows the better performance in recognition of genuine user using weighted fusion compared to Bayesians fusion. Score level fusion using weighted sum and Bayesian achieved less False Acceptance of imposters compared to individual finger knuckle or finger vein.

The complexity of the proposed algorithm is summarized a under. Let $\mathrm{N}$ represent total number of user and each user has $\mathrm{M}$ samples. Let $\mathrm{M} 1$ and $\mathrm{M} 2$ represents number of test and training samples used such that $M 1+M 2=M$. So we select $(M 1 \times N)$ test samples and $(M 2 \times N)$ samples. For verification, each test sample Ti ( $T i$ for $i=1$ to 3 ) is compared with all training samples $\operatorname{Trj}$ ( $\operatorname{Trj}$ for $j=1$ to 7 ), So for one user matching complexity is $\mathrm{O}(\mathrm{M} 1 \mathrm{M} 2)$ and for $\mathrm{N}$ user $\mathrm{O}(\mathrm{M} 1 \mathrm{M} 2 \times \mathrm{N})$.

For identification, ( $\mathrm{N} \times \mathrm{M} 1$ ) test samples and (NX M2) training samples are considered. So matching complexity is $\mathrm{O}[\mathrm{N}(\mathrm{N}-1) \times \mathrm{M} 1]$ for each biometric. Using conventional matching the complexity is $\mathrm{O}[\mathrm{N}$ (N-1) X M1 M2]. For multimodal biometric using FK and FV, matching complexity is $\mathrm{O} 2[\mathrm{~N}(\mathrm{~N}-1) \times \mathrm{M} 1]$. For $\mathrm{N}=50$ users and $\mathrm{M} 1=3$ (test samples) and $\mathrm{M} 2=7$ (training samples), complexity using conventional method is 51450 tests need to perform. Proposed algorithms performed 7350 tests for matching, which shows great improvement in time complexity. However the complexities of algorithms depend on number of features used to represents the samples in the database. If each sample is represented by $P$ features, then the complexity of the algorithm is $O[P \times N(N-1) \times M 1]$. In the proposed multimodal recognition algorithm, 500 finger knuckle and 500 finger vein samples are used. For fusion at score level using weighted sum, requires 500 additions and 1000 multiplications. In general, number of additions and multiplication required would be $\mathrm{N}$ and $2 \mathrm{~N}$.

\section{Results and Conclusion}

Proposed multimodal biometric authentication integrates transformed finger knuckle and finger vein features using score level fusion. To demonstrate the efficiency recognition, experiments based on own finger knuckle and finger vein database performed. The finger knuckle and finger vein features are successfully integrated using Bayesian and weighted sum method. The recognition accuracy of integrated biometric is improved as compared to individual finger knuckle and finger vein. The fusion of these two modalities using Bayesian method demonstrated the recognition accuracy of $98.3 \%$. Various weights of finger knuckle and finger vein affects the recognition accuracy. The better recognition accuracy is obtained at weight of finger knuckle is 0.8 and finger vein is 0.2 . The performance index is improved i.e. $98.5 \%$ and the Error Equal Rate is $1.5 \%$. The multimodal biometric recognition accuracy improved by these two fusion methods. Error equal rate is reduced by $10 \%$ than individual biometric system. The weighted sum fusion reduces error equal rate by $0.2 \%$ as compared to Bayesian method. However multimodal system has the limitations of high setup cost, high computational complexity and non-standard classification rule. In the proposed algorithm, matching complexity is $\mathrm{O}\left[\mathrm{N}(\mathrm{N}-1) \times \mathrm{M}_{1}\right]$ for each biometric. Using conventional matching the complexity is $\mathrm{O}\left[\mathrm{N}(\mathrm{N}-1) \times \mathrm{M}_{1} \mathrm{M}_{2}\right]$. For multimodal biometric using $\mathrm{FK}$ and $\mathrm{FV}$, matching complexity is $\mathrm{O} 2\left[\mathrm{~N}(\mathrm{~N}-1) \times \mathrm{M}_{1}\right]$. It shows great reduction in matching complexity using the proposed algorithms.

\section{REFERENCES}

[1] Cheng-BoYu, Quin, LianZhang, Yan-Zhe Cui ,"Finger vein Image Recognition Combining Modified Hausdorff distance with minutia feature matching", Biomedical Science and Engineering. 2009

[2] Naoto Miura, Akio Nagasaka, Takafumi Miyatake, "Extraction of Finger-Vein Patterns Using Maximum Curvature Points in Image Profiles", IAPR Conference on Machine Vision Applications, Tsukuba Science City, Japan. 2005, 
[3] Arun Ross, Anil k. Jain, "Multimodal Biometrics: An Overview", Proc. Of $12^{\text {th }}$ European Signal Processing Conference (EUSIPCO), (Vienna Austria), September 2004, pp. 1221-1224.

[4] [20] L.Hong, A.K. Jain, and S. Pankanti, "Can Multibiometrics improves performance?" in Proc. AutolD'99, Summit, NJ, USA. pp. 59 - 64.

[5] Zhang, Lei Zhang, David Zhang and Hailong Zhu, “Online Finger-Knuckle-Print Verification for Personal Authentication", Biometrics Research Center, Department of computing, The Hong Kong Polytechnic University

[6] M. Indovina, U. Uludag, R. Snelick, A. Mink, A. Jain, "Multimodal Biometric Authentication Methods: A COTS Approach", National Institute of Standards and Technology, Michigan State University, Appeared in Workshop on MMUA, December 2003.

[7] Ajay Kumar, Ch. Ravikanth, "Personal Authentication using Finger Knuckle Surface”, IEEE Transactions on Information Forensics and Security, vol. 4, no. 1, March. 2009, pp. 98-110,

[8] Lin Zhang, Lei Zhang, David Zhang, Zhenhua Guo, "Phase Congruency induced Local features for Fi nger-knuckle-print Recognition", Patten Recognition,45, 2012, pp. 2522-2531.

[9] Michael K.O. Goh, Connie Tee, Andrew B.J. Teoh “BI-modal Palm Print And Knuckle Print Recognition System", Journal of IT in Asia, vol 3, (2010). (FKP4)

[10] Shrotri A., Rethrekar S.C., Patil, M.H. Debnath, Bhattacharyya, Tai-hoonKim, "Infrared Imaging of Hand Vein Pattern for Biometric Purpose", Journal of Security Engineering2009.

[11] Naoto Miura, Akio Nagasakat, Takafumi Miyataket, “Automatic Feature Extraction from non-uniform Finger Vein Image and its Application to Personal Identification", IAPR Workshop on Machine Vision Applications, Japan, 2002 .

[12] Dana Hejitmankova, Radim Dvorak, Martin Drahansky, and Filip Orsag, "A new method of Finger Vein Detection", International journal of Bio-science and Biotechnology, 1(1) , 2009

[13] Laxmi C., Deepaka, Kandaswamy A., , "An Algorithm For Improved Accuracy in Unimodal Biometric Systems through Fusion of Multiple Features Sets" ,ICGST-GVIP 9(III), 2009

[14] Jing Zhang, Jinfeng Yang, , " Finger-Vein Image enhancement Based on Combination of Gray-Level Grouping and Circular Gabor Filter ", IEEE,2009

[15] Etta D., Pisano, Shuquan Zoog, Bradley m. Hemminger, Marla DeLuca, R. Eugene Johnston, Keith Muller M. Patricia Braeuning, and Stephen Pizer, “Contrast Limited Adaptive Histogram Equilization Image processing to improve the Detection of Simulated Speculation in dense Mammograms", Journal of Digital Imaging, 11 (4) , 1998

[16] Stephin M. Pizer,E. Philip Amburn, John D. Austin,Robert Cromartie, Ari Geselowtiz, Trey Greer,Bart Ter Haar Romeny, John B. Zimmerman, and Karel Zuiderveld, "Adaptive histogram Equalization and its Variations", Computer Vision, Graphics, and Image Processing, 39,355-368, ,

[17] Tomasi C., Manduch R., "Bilateral Filtering for Gray and Color Images", in Proceedings of IEEE International Conference on Computer Vision, Bombay, India, 1998, pp 237-240 
Sujata Kulkarni and Ranjana Raut; Wavelet Based Finger Knuckle and Finger Vein Authentication System. Advances in I mage and Video Processing, Volume 4 No 4, August (2016); pp: 1-16

[18] H.B.Kekre and V.A.Bharadi, "Finger Knuckle Verification using Kekre's Wavelet Transforms", International Conference \& Workshop on Emerging Trends in Technology, ACM Digital Library, New York; 2011. pp 32-37,

[19] H. B. Kekre, Archana Athawale, Dipali Sadavarti, "Algorithm to Generate Wavelet Transform from an Orthogonal Transform”, International Journal of Image Processing; 2011.

[20] Sujata Kulkarni, Ranjana Raut and Pravin Dakhole, "Wavelet Based Modern Finger Knuckle Authentication", Accepted for publication Published by Elsevier B.V in proceedings 4 International Conference on Eco-friendly Computing and Communication Systems, NIT Kurusetra, India, Elsevier Publications Science Direct Procedia Computer science.

[21] Sujata Kulkarni, Ranjana Raut and Pravin Dakhole "A Novel Authentication System Based on Hidden Biometric Trait" Accepted for publication in proceedings of International Conference on Computational Modeling and Security (CMS 2016), Banglore, Elsevier Publications, Science Direct Procedia Computer science.

[22] Feifei CUI and Gongping Yang, "Score Level Fusion of Fingerprint and Finger Vein Recognition", Journal of Computational Information Systems 7: 16 (2011) 5723-5731 